Ann. Zootech., I97I, $20(2)$, I35-I52.

\title{
INFLUENCE, CHEZ LE TAURILLON EN CROISSANCE, DU TRAITEMENT DE GONSERVATION (ENSILAGE OU DÉSHYDRATATION) DE LA CÉRÉALE IMMATURE (ORGE, MAÏS) SUR SA VALEUR NUTRITIVE ET SUR L'EFFICACITÉ MÉTABOLIQUE DE L'URÉE AJOUTÉE (1)
}

\author{
S.-Z. ZELTER, Geneviève CHARLET-LERY et J.-L. TISSERAND ( $\left.{ }^{2}\right)$ \\ avec la collaboration technique de F. Le Descirault de Monredon et Christiane Dumay \\ Laboratoire de Recherches sur la Conservation et l'Efficacité des Aliments, \\ Centre national de Recherches zootechniques, I.N.R.A., \\ 78 - Jouy-en-Josas
}

\section{RÉSUMÉ}

Au cours de 4 expériences exécutées successivement sur 44 taurillons on a étudié l'effet de la conservation (ensilage, déshydratation) de deux céréales immatures (orge et mais) sur:

a) la digestibilité et la valeur nutritive de ces aliments ;

b) l'utilisation métabolique de leurs protéines ;

c) l'utilisation métabolique comparée de l'azote d'urée et de tourteau de soja.

Deux récoltes successives (1967 et I968) d'orge (variété Rika) en coupe haute (I5-30 cm sous l'épi) et l'épi entier de maïs (variété $I . N . R . A .260$ ) ont été effectuées $2-4$ semaines avant maturité normale du grain, et traitées soit par ensilage sous vide partiel, soit par déshydratation en séchoir à basse température ( $120-130^{\circ} \mathrm{C} \times 30-40 \mathrm{mn}$.). Les mesures de la digestibilité et de la rétention azotée ont été effectuées selon un dispositif en carré latin ou de blocs comparables (tabl. I). Il a été constaté que :

a) le produit déshydraté et condensé possède une digestibilité plus basse que le produit ensilé haché ; sa valeur énergétique serait apparemment plus faible (tabl. 5) ;

b) les coefficients de rétention (CR) et d'utilisation pratique (CUP) de l'azote sont plus élevés après déshydratation qu'après ensilage. La supériorité du produit déshydraté serait imputable à sa très faible teneur en $\mathrm{N}$ soluble (tabl. $6,7,8)$;

c) l'utilisation métabolique de l'azote de l'uréc est améliorée lorsque celle-ci supplémente la céréale déshydratée : elle est égale (maïs) voire même supérieure (orge) à celle de l'azote du soja. Avec la céréale ensilée l'urée est moins efficace que le tourteau de soja (tabl. 6, 7, 8) ;

d) l'action bénéfique de la déshydratation sur l'utilisation de l'azote uréique se relierait à la forte augmentation de la solubilité amylolytique ( $\alpha$-amylase) de l'amidon induite par le traitement thermique expérimenté (tabl. 4 et fig. I).

(1) Recherche poursuivie dans le cadre d'une action concertée intra INRA. et D.G.R.S.T.

(') Adresse actuelle : E. N. S. A. A., boulevard du Docteur-Petitjean, 2 I - Dijon. 


\section{INTRODUCTION}

Une technique récente consiste à récolter l'épi entier de céréale avec une partie plus ou moins haute de sa tige, au stade laiteux et, de préférence, cireux-vitreux du grain soit environ 2-3 semaines avant maturité complète, pour alimenter des ruminants ou des porcs. En comparaison avec une récolte de grain mûr, cette technique permettrait, selon ses promoteurs, d'augmenter sensiblement la production de matière sèche et d'unités fourragères à l'hectare et d'effectuer une culture dérobée dans des régions où les conditions climatiques le permettent.

La teneur en eau d'une céréale ainsi récoltée avoisine 40-50 p. Ioo et sa conservation par ensilage ou par déshydratation est aisée (Goust et al., I97I). Le premier de ces traitements occasionne des pertes limitées de matière nutritive, dégrade la plus grande part d'amidon facilement attaquable en acides organiques (acides lactique et gras volatils) et augmente la teneur en azote soluble total et ammoniacal ; le second qui n'occasionne ni pertes nutritives, ni modification sensible de la fraction azotée, est susceptible dans certaines conditions d'humidité et detempérature d'accrô̂tre la solubilité de l'amidon et sa sensibilité amylolytique (GuIlbot et MERCIER I962).

Une céréale immature traitée de la sorte, devrait pouvoir couvrir, chez le taurillon en croissance, la quasi-totalité de ses besoins énergétiques. Mais sa valeur nutritive exacte n'est pas connue.

Le rapport MAD/UF de cet aliment variant entre 60 et 90 , alors que le taurillon en croissance intensive en exige selon les normes environ $\mathrm{I} 2 \mathrm{O}$, 1'autre problème posé est celui de sa supplémentation azotée. L'emploi de tourteaux classiques (soja, arachide) devient de plus en plus coûteux; l'urée pourrait peut-être les remplacer, mais on ignore si elle a une efficacité satisfaisante pour complémenter une céréale immature, ensilée ou déshydratée.

De nombreux facteurs encore mal définis règlent effectivement la vitesse et l'amplitude de la protéinosynthèse microbienne dans la panse à partir d'azote non protéique. Parmi ceux-ci figurent sans aucun doute la concentration en $\mathrm{N}-\mathrm{NH}^{3}$ (LEwIS, I96r) et le pH du rumen (Chalmers, I969), la capacité de stockage d'ions ammonium par les bactéries du rumen (Hoshino et al., I966), l'énergie libérée par la fermentation (BLOMFieid et al., I964; HunGaTe, I966).

La disponibilité de l'énergie semble un élément prépondérant : la présence d'une source d'énergie hautement assimilable dans le rumen, y stimule la protéinogenèse bactérienne (Kameoka et al., I962 ; Hoover et al., I963). Ainsi des moutons consommant des rations riches en urée et contenant suffisamment de glucides disponibles, fixent davantage d'ammoniaque que ceux recevant un régime normal (McLARREN, Ig6r et I966).

L'amidon considéré comme une source particulièrement favorable à l'utilisation de l'urée dans le rumen (Dyck, I963), assurerait une libération persistante d'énergie à un niveau suffisamment élevé.

L'origine botanique de l'amidon jouerait un rôle important. Ainsi lorsqu'il provient du maïs, il n'a pas été observé, chez la vache laitière, de différence d'efficacité entre urée et tourteau de soja ; mais lorsqu'il provient d'un mélange d'avoine 
et de tapioca, l'efficacité de l'urée n'était plus que 52 p. Ioo de celle du soja (MöLLER, et al., I967 et I968).

L'on peut se demander si une modification technologique de l'état de l'amidon n'interviendrait pas également.

En admettant que la déshydratation puisse augmenter sensiblement dans une céréale la solubilité de son amidon, elle devrait faciliter également son attaque par la micropopulation amylolytique du rumen. L'ensilage qui, au contraire, dégrade l'amidon en acides organiques priverait la micropopulation ruminale de cette source adéquate d'énergie indispensable à sa croissance.

Afin d'élucider ces divers points nous avons étudié l'influence de ces deux procédés de conservation appliqués à deux céréales immatures (orge et maïs) sur leur valeur alimentaire propre et sur la rétention de l'azote uréique chez le taurillon en croissance. Quelques résultats de cette étude ont été rapportés antérieurement (Zeiter et Charlet-Lery, ig69; Zelter et al., I969).

\section{I. - MATÉRIEI, ET MÉTHODES}

$$
\text { I. L Les animaux }
$$

Des taurillons de race nomande âgés d'environ 6-7 mois pesant entre 200 et $250 \mathrm{~kg}$ ont été répartis en groupes homogènes de 2 ou 3 sujets et affectés suivant une répartition au hasard aux divers régimes.

$$
\text { 2. - Dispositifs expérimentaux }
$$

Quatre expériences successives ont été effectuées soit selon un dispositif en carré latin, soit selon celui des blocs comparables avec régimes interchangeants. Elles se sont déroulées :

- de novembre 1967 à mars 1968 pour l'orge récoltée en juillet I967;

- d'avril I968 à juillet I968 pour le maîs récolté en octobre 1967;

- đe janvier I 969 à mai I969 pour l'orge récoltée en juillet I968;

- d'octobre I969 à janvier I970 pour le maïs récolté en octobre I 968.

Le tableau $x$ indique les divers schémas expérimentaux utilisés successivement et les sigles désignant les régimes.

Les animaux séjournaient en stalles individuclles à bilan permettant la collecte séparée des fèces et de l'urine. Le modèle initial trop surélevé entraînant une fatigue des animaux a obligé à réduire la durée de l'expérience de 3 (orge 1967) à 2 périodes (mäis I967) et à remplacer le carré latin par un dispositif de blocs comparables.

Pour la récolte r968, des modifications ont été introduites dans le modèle des stalles qui assuraient une meilleure adaptation des animaux à leur milieu. Cela a permis d'effectuer une troisième période avec l'orge, et prolonger la durée des période de mesure avec le maïs. De ce fait la longueur des périodes de bilan variait d'un essai à l'autre.

Chaque période comportait une phase d'accoutumance d'un mois au minimum, dont une partie au sol, et une phase de mesures de ro jours (orge et maïs I967), de I5 jours (orge Ig68) ou 2 fois Io jours (maïs I968).

$$
\text { 3. - Les aliments }
$$

En 1967 et I968 l'orge (variété $R i k a$ ) et le maîs (variété $I N R A$ 26o) ont été récoltés avant maturité, avec une teneur en matière sèche (MS) comprise entre 45 et 52 p. Ioo. L'orge fauchée en coupe haute ( $15 \mathrm{~cm}$ en 1967 et $30 \mathrm{~cm}$ environ en 1968 au-dessous de la base du rachis), a été hachée, mais la paille est restée en brins plus longs en I967 qu'en 1968. L'épi entier de maïs (spathes incluses) a subi aussitôt cueilli, un double broyage. Les caractéristiques des céréales au moment de la récolte sont indiquées dans un autre mémoire (Gouer et al., I97I). 
TABLEAU I

Schéma du dispositif expérimental

\begin{tabular}{|c|c|c|c|c|}
\hline & & Orge 1967 & sujets & lot) \\
\hline & Lot 1 & Lot 2 & Lot 3 & \\
\hline & - & - & - & \\
\hline $1^{\text {re }}$ période & OEU & ODU & OETS & \\
\hline $2^{e}$ période & ODU & OETS & OEU & \\
\hline $3^{e}$ période & OETS & OEU & ODU & \\
\hline & & Orge 1968 & sujets & lot) \\
\hline & $\begin{array}{c}\text { Lot } \\
-\end{array}$ & $\begin{array}{c}\text { Lot } 2 \\
-\end{array}$ & $\begin{array}{c}\text { Lot } 3 \\
-\end{array}$ & Lot \\
\hline $1^{\text {re }}$ période & OEU & $\operatorname{OEUP}\left({ }^{*}\right)$ & OETS & ODU \\
\hline $2^{\mathrm{e}}$ période & OEUP & OEU & ODU & OETS \\
\hline $3^{e}$ période & & & & \\
\hline
\end{tabular}

1. Mä̈s 1967 (3 sujets par lot)

$\begin{array}{lllll} & \text { Lot } 1 & \text { Lot } 2 & \text { Lot } 3 & \text { Lot } 4 \\ 1^{\text {re période }} & \text { METS } & \text { MEU } & \text { MDTS } & \text { MDU } \\ 2^{\text {e période }} & \text { MEU } & \text { METS } & \text { MDU } & \text { MDTS }\end{array}$

2. Maïs 1968 (2 ou 3 sujets par lot)

$\begin{array}{lcccccc} & \text { Lot } 1 & \text { Lot } 2 & \text { Lot } 3 & \text { Lot } ~_{4} & \text { Lot } 5 & \text { Lot } 6 \\ 1^{\text {re période }} & \text { METS } & \text { MDTS } & \text { MDU } & \text { MD } & \text { MEU } & \text { ME } \\ 2^{\mathrm{e}} \text { période } & \text { MDTS } & \text { METS } & \text { MD } & \text { MDU } & \text { ME } & \text { MEU }\end{array}$

Sigles désignant les régimes :

- Orge : $\mathrm{OE}=$ orge ensilée $; \mathrm{OD}=$ orge déshydratée ; $\mathrm{OEU}=\mathrm{OE}+$ urée $; \mathrm{OEUP}=\mathrm{OE}+\mathrm{urée}$ protégée ; ODTS $=$ OD + tourteau de soja.

- Maïs : $\mathrm{ME}=$ maïs ensilé $; \mathrm{MD}=$ maïs déshydraté $; \mathrm{MlU}=\mathrm{ME}+$ urée $;$ METS $=\mathrm{ME}+$ tourteau de soja; $\mathrm{MDU}=\mathrm{MD}+$ urée $;$ MDTS $=$ MD + tourteau de soja.

$\left(^{*}\right)$ Il ne sera pas discuté ici de la comparaison urée naturelle, urée protégée (OEU $\leftrightarrow$ OEUP).

$1=$ récolte $1967 ; 2=$ récolte 1968 .

Dans tous les cas, la même matière première a été traitée au même moment :

- Soit par ensilage : orge et maïs ont été conservés sous vide partiel dans des silos en butyl de $4 \mathrm{~m}^{3}$ maintenus rigides par une armature de grillage. Aucun conservateur n'a été ajouté.

Avant utilisation, soit après 5 à 8 mois de conservation tous les silos ont été sondés pour en déterminer la qualité. Les résultats analytiques donnés au tableau 2 montrent le très bon état des ensilages. Certains d'entre eux ont été enrichisen azote, soit par incorporation d'urée au moment de la mise en silos, soit par addition de tourteau de soja au moment de leur distribution à l'auge.

- Soit par déshydratation à basse température dans un séchoir Scolari. Les températures de séchage et les durées ont été pour :

- l'orge 1967 et 1968: 12 $5^{\circ}-130^{\circ} \mathrm{C}, 20$ à 30 minutes;

- le maïs 1967 : $135^{\circ} \mathrm{C}$, 30 à 35 minutes, un second passage des rafles insuffisamment sèches dans la déshydrateuse a été nécessaire, ce qui porte cette durée à environ 60 minutes;

- le maïs I 968 : $130^{\circ} \mathrm{C}$, 60 minutes.

Les céréales déshydratées ont été broyées à la grille to $\mathrm{mm}$, additionnées éventuellement d'urée ou de tourteau de soja, et agglomérées en bouchons de $7 \mathrm{~mm}$ de diamètre. 
TABLEAU 2

Analyse qualitative moyenne des ensilages consommés par les animaux durant les périodes de mesure

\begin{tabular}{|c|c|c|c|c|c|c|c|}
\hline & \multirow{2}{*}{$\mathrm{pH}$} & \multicolumn{6}{|c|}{$\mathrm{g} / \mathrm{kg}$ de frais } \\
\hline & & $\begin{array}{l}\mathrm{N}-\mathrm{NH}_{3} \\
\% \mathrm{~N} \text { tot. }\end{array}$ & $\begin{array}{c}\text { Acide } \\
\text { lactique }\end{array}$ & $\begin{array}{c}\text { Acide } \\
\text { acétique }\end{array}$ & $\begin{array}{c}\text { Acide } \\
\text { propionique }\end{array}$ & $\begin{array}{c}\text { Acide } \\
\text { butyrique }\end{array}$ & Alcool \\
\hline \multicolumn{8}{|l|}{ Orge } \\
\hline $\mathrm{OE}_{1}$ & 4,65 & 5,1 & 6,68 & $1,7 \geq$ & 0,28 & 2,82 & 7,18 \\
\hline $\mathrm{OEU}_{1}$ & 5,56 & 30,1 & 10,78 & 4,34 & 0,66 & 4,51 & 6,57 \\
\hline $\mathrm{OE}_{2}$ & 5,84 & 4,6 & 6,19 & 1,81 & 0,18 & 1,67 & 5,84 \\
\hline $\mathrm{OEU}_{2}$ & 4,73 & 20,8 & 8,84 & $3,7:$ & 0,22 & 1,56 & 4,72 \\
\hline \multicolumn{8}{|l|}{ Maïs } \\
\hline $\mathrm{ME}_{1}$ & 4,7 & 3,6 & 9,90 & 2,89 & - & - & 4,58 \\
\hline $\mathrm{MEU}_{\mathrm{t}}$ & 5,8 & 13,2 & 13,70 & 3,17 & 0,77 & 0,55 & 6,61 \\
\hline $\mathrm{ME}_{2}$ & 4,2 & 9,6 & 13,67 & 4,17 & 0,18 & 0,21 & 2,41 \\
\hline $\mathrm{MEU}_{2}$ & 4,4 & 10,7 & 16,05 & 4,32 & 0,19 & 0,79 & 3,45 \\
\hline
\end{tabular}

$1=$ récolte $1967 ; 2=$ récolte 1968.

\section{4. - Les régimes et le rationnement}

Afin d'élever la teneur en $\mathrm{N}$ des rations à base de céréales au niveau des besoins d'un taurillon en croissance (environ I $20 \mathrm{~g} \mathrm{MAD} / \mathrm{UF}$ ), deux sources d'azote ont été expérimentées comme déjà dit : le tourteau de soja et l'urée (urée technique à 42,4 p. Ioo N).

Les quantités de ces suppléments ajoutées aux produits ensilés ou déshydratés ont été calculées à partir d'analyses préliminaires des matières premières. Pour l'urée, elles étaient de l'ordre de $13 \mathrm{~g} / \mathrm{kg}$ de MS d'orge et de $23 \mathrm{~g} / \mathrm{kg}$ de MS de maîs ; pour le tourteau de soja, les quantités respectives atteignaient environ $80 \mathrm{~g}$ et $\mathrm{I}_{5} 6 \mathrm{~g}$.

L'ingestion de MS a été réglée en fonction. du poids métabolique moyen $\left(\mathbf{P}^{0,75}\right)$ supposé à chaque mi-période de bilan. Pour chaque expérience, ce niveau a été rajusté dans tous les groupes supplémentés en azote sur celui des plus faibles consommateurs, afin d'égaliser les apports d'énergie et d'azote de chacun des régimes.

Les régimes à céréales non supplémentés ont été distribués, par contre, ad libitum.

L'orge 1967 et les 2 maîs (I967 et 1968) trop pauvres en cellulose brute, ont été additionnés de paille de blé (8-Io p. Ioo) distribuée séparément sous forme hachée, afin de porter le taux cellulosique au voisinage de I2-I 5 p. Ioo.

Les animaux recevaient hebdomadairement une supplémentation vitaminique $A$ et $D$, et disposaient ad libitum de pierres à lécher complètes d'origine commerciale.

\section{5. - Collecte, conservation et analyse des prélèvements}

L'urine était collectée en milieu acidifié à l'acide sulfurique ( Io ml $\mathrm{SO}_{4} \mathrm{H}_{2}$ à 25 p. roo/litre d'urine) que l'on pulvérisait d'autre part 2 fois par jour sur les fèces pour éviter toutefermentation.

Des prélèvements journaliers aliquots frais de fèces d'urine et d'ensilage ont été conservés $\grave{a}+I^{\circ} \mathrm{C}$, et des dosages d'azote ont été effectués par la méthode Kjeldahl sur chaque échantillon moyen pondéré de 5 jours. 
D'autres prélèvements journaliers d'aliments et de fèces ont été séchés dans une étuve à $70^{\circ} \mathrm{C}$, afin de constituer en fin de période des échantillons moyens cumulés sur lesquels ont été déterminées les matières minérales et cellulosiques (cellulose Weende).

L'amidon total, la solubilité amylolytique (test à la $\alpha$-amylase) et les quantités totales d'amidon solubilisé dans les céréales à la récolte et après traitement de conservation ont été déterminés par la méthode de Guilbot et Mercier (I962).

\section{II. - RÉSULTATS}

L'état général des animaux était très bon à tout moment.

L'addition de paille hachée à tous les régimes a assuré aux taurillons le minimum de particules grossières longues qui ont permis d'éviter des troubles digestifs (diarrhée, arrêt de rumination) observés lors d'un court essai préliminaire.

La composition des rations effectivement consommées figure dans le tableau 3 ; la teneur des céréales en amidon total et les résultats des tests amylasiques sont donnés dans le tableau 4 et la figure $\mathrm{I}$.

TABLEAU 3

Composition des rations effectivement consommées (en p. Ioo MS)

\begin{tabular}{|c|c|c|c|c|c|c|c|}
\hline & \multicolumn{3}{|c|}{ Orges } & & \multicolumn{3}{|c|}{ Maïs } \\
\hline & $\begin{array}{c}\text { Matières } \\
\text { organiques }\end{array}$ & $\begin{array}{l}\text { Matières } \\
\text { azotées }\end{array}$ & $\begin{array}{l}\text { Cellulose } \\
\text { Weende }\end{array}$ & & $\begin{array}{l}\text { Matières } \\
\text { organiques }\end{array}$ & $\begin{array}{l}\text { Matières } \\
\text { azotées }\end{array}$ & $\begin{array}{l}\text { Cellulose } \\
\text { Weende }\end{array}$ \\
\hline $\mathrm{ODU}_{1}$ & 94,6 & 10,5 & 13,1 & $\mathrm{MDU}_{1}$ & 97,1 & 12,9 & 10,7 \\
\hline $\mathrm{OEU}_{1}$ & 94,2 & 11,5 & 14,2 & MDTS $_{1}$ & 96,8 & 12,6 & 10,6 \\
\hline $\mathrm{OETS}_{1}$ & 94,7 & 12,4 & 12,0 & $\mathrm{MEU}_{1}$ & 97,7 & 11,2 & 12,4 \\
\hline & & & & METS $_{1}$ & 96,2 & 12,8 & 11,0 \\
\hline $\mathrm{ODU}_{2}$ & $9 \div, 9$ & 13,8 & 11,8 & $\mathrm{MDU}_{2}$ & 97,4 & 16,4 & 10,9 \\
\hline $\mathrm{OEU}_{2}$ & 93,9 & 12,8 & 15,3 & MDTS $_{2}$ & 97,0 & 16,7 & 9,8 \\
\hline $\mathrm{OETS}_{2}$ & $9_{4,2}^{\prime}$ & 12,7 & 14,3 & $\mathrm{MEU}_{2}$ & 97,7 & 16,7 & 12,7 \\
\hline $\mathrm{OE}_{2}$ & 94,3 & 9,6 & 15,2 & METS $_{2}$ & 96,9 & 16,4 & 11,7 \\
\hline $\mathrm{OD}_{2}$ & 94,0 & 10,6 & 12,1 & $\mathrm{ME}_{2}$ & 97,8 & 8,1 & 13,2 \\
\hline & & & & $\mathrm{MD}_{2}$ & 97,9 & 8,6 & 11,1 \\
\hline
\end{tabular}

$1=$ récolte $1967 ; 2=$ récolte 1968.

\section{I. - Niveau de consommation de matières séches (tab1. 5)}

Le rationnement égalisé n'a pas permis de tester 1'effet des traitements de conservation sur l'acceptabilité de la céréale immature supplémentée en azote. Les niveaux d'ingestion les plus élevés ont été néanmoins enregistrés pour les céréales supplémentées en azote. Excepté l'essai maïs I967, les niveaux sont assez semblables dans chaque série expérimentale. 
TABLEAU 4

Caractéristiques de l'état de l'amidon

\begin{tabular}{|c|c|c|c|c|c|c|c|c|c|c|c|c|}
\hline & \multicolumn{4}{|c|}{ Amidon total p. $100 \mathrm{MS}$} & \multicolumn{4}{|c|}{$\begin{array}{c}\text { g d'amidon facilement } \\
\text { attaquable à l'a-amylase } \\
\text { par kg de MS }\end{array}$} & \multicolumn{4}{|c|}{$\begin{array}{l}\text { Rapport amidon facilement } \\
\text { attaquable/urée incorporée }\end{array}$} \\
\hline & \multicolumn{2}{|c|}{ Orge } & \multicolumn{2}{|c|}{ Maïs } & \multicolumn{2}{|c|}{ Orge } & \multicolumn{2}{|c|}{ Maîs } & \multicolumn{2}{|c|}{ Orge } & \multicolumn{2}{|c|}{ Maîs } \\
\hline & 1967 & 1968 & 1967 & 1968 & 1967 & 1968 & 1967 & 1968 & 1967 & 1968 & 1967 & 1968 \\
\hline Céréale originelle & 43,0 & 34,1 & 47,1 & 44,5 & 66 & 45 & 46 & 20 & $(5,0)$ & $(3,5)$ & $(2,0)$ & $(0,91)$ \\
\hline $\begin{array}{l}\text { Céréale ensilée } \\
\quad+\text { urée }\end{array}$ & 41,2 & 34,3 & - & 51,8 & 31 & 32 & 9,5 & 16 & 2,5 & 2,5 & 0,4 & 0,70 \\
\hline $\begin{array}{l}\text { Céréale déshy- } \\
\text { dratée + urée }\end{array}$ & 41,9 & 29,8 & 50,8 & 50,6 & 325 & 206 & 128 & 48 & 25 & 15,9 & 6,0 & 2,2 \\
\hline
\end{tabular}

(1) Urée incorporée par $\mathrm{kg}$ MS : $13 \mathrm{~g}$ pour l'orge ; $23 \mathrm{~g}$ pour le maïs.

( ) Les valeurs entre parenthèses résultent d'un calcul théorique.

\section{2. - Gain de poids}

Les performances de croissance réalisées sur des courtes périodes (6-8 semaines) par des animaux en cage à bilan ne peuvent pas être considérées comme critère d'efficacité d'un régime alimentaire. Les gains quotidiens notés n'ont qu'une valeur indicative ; ils sont relativement faibles (479 à 73I g avec les régimes d'orge, 5I9 à I Ir3 g avec les régimes maîs) et s'expliquent par les bas niveaux d'ingestion d'énergie.

\section{3. - Coefficients d'utilisation digestive (CUD) (tab1. 5)}

Les teneurs en cellulose (tabl. 3) des régimes expérimentés au cours de chaque expérience sont assez proches : I2 à I5 p. Ioo avec l'orge, to à I3 p. Ioo avec le maïs. Les régimes déshydratés renferment d'après l'analyse I à 3 points moins de cellulose que les régimes ensilés. La même matière première ayant servi aux deux traitements technologiques, l'écart observé est sans doute dû à des difficultés d'échantillonnage de l'ensilage.

Par rapport à la forme longue ensilée, la forme déshydratée condensée abaisse les CUD de la MS et de la matièreorganique (MO), quelle que soitla céréale. Cette chuteest significative et plus forte en l'absence qu'en présence de supplémentation azotée. Les CUD-MO des régimes supplémentés sont en moyenne de : 67,4 pour OEU et 66,6 pour OETS contre 65,4 pour ODU, 7 I , 8 pour MEU contre 69,0 pour MDU, 73,6 pour ME'TS contre 70,o pour MDTS. La matière organique des régimes à base de maîs est systématiquement plus digestible que celle des régimes à base d'orge. Dans tous les cas, malgré la dispersion des données, la forme déshydratée possède des CUD de cellulose Weende nettement plus faibles que la forme ensilée. La chute varie entre I2 et $3^{8}$ points. 


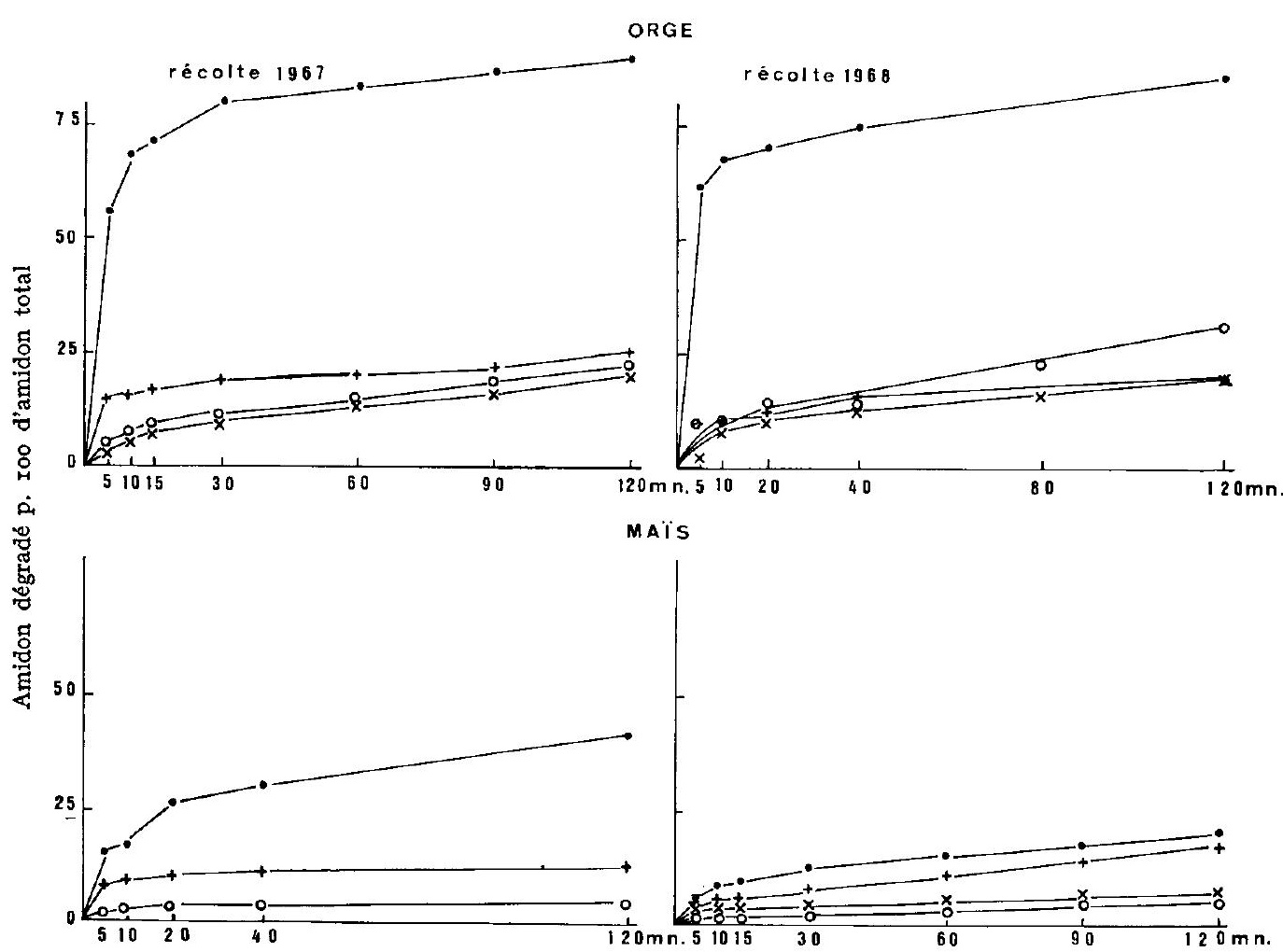

FIG. I. - Cinétique de l' $\alpha$-amylolyse in vitro

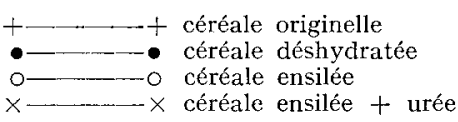

Le mode de présentation de la céréale et la nature du complément azoté ne modifient pas le CUD apparent de l'azote (CUDN) ; par contre, la présence du complément l'améliore notablement. L'amélioration atteint II points pour les régimes d'orge (64 contre 53 ) et 18 points pour ceux de mais (7o contre 52 ).

\section{4. - Énergie et matières azotées digestibles (tabl. 5).}

La céréale étant toujours complémentée en cellulose ou à la fois en cellulose et en azote, les calculs sont faits pour les rations globales telles qu'ingérées.

Les valeurs nutritives sont exprimées en MOD/kg MS, qui reflète 1'énergie digestible.

En l'absence de complémentation azotée, la forme déshydratée apporte dans le cas de l'orge, 9 p. roo de moins, et dans celui du mais 6 p. Ioo de moins d'énergie digestible que la forme ensilée.

En présence du complément azoté, la différence s'atténue nettement pour l'orge $(-2$ p. IOO) et est à peine mođifiée pour le maĩs $(-5$ p. IOO) 
TABLEAU 5

Coefficients de digestibilité et valeur nutritive des régimes

\begin{tabular}{|c|c|c|c|c|c|c|c|}
\hline & $\begin{array}{l}\text { MSI } \\
/ P^{0,75}\end{array}$ & $\begin{array}{l}\text { Matières } \\
\text { sèches }\end{array}$ & $\begin{array}{c}\text { Matières } \\
\text { organiques }\end{array}$ & $\begin{array}{l}\text { Matières } \\
\text { azotées }\end{array}$ & $\begin{array}{l}\text { Cellulose } \\
\text { Weende }\end{array}$ & $\begin{array}{c}\text { MOD } \\
\text { (g/kg MS) }\end{array}$ & $\begin{array}{c}\text { MAD } \\
\text { (g/kg MS) }\end{array}$ \\
\hline \multicolumn{8}{|l|}{ Orge } \\
\hline $\mathrm{OF}_{2}$ & $86 \pm 3$ & $63,0 \pm 0,1$ & $66,0 \pm 0,4$ & $51,8 \pm 0,6$ & $1^{\prime} \pm 2$ & 622 & 56 \\
\hline $\mathrm{OD}_{2}$ & $84 \pm 2$ & $58,1 \pm 1,4$ & $60,6 \pm 1,2$ & $53,6 \pm 1,7$ & $6 \pm 2$ & 570 & 51 \\
\hline $\mathrm{OEU}_{1}$ & $76 \pm 4$ & $65,1 \pm 1,0$ & $67,9 \pm 1,0$ & $65,7 \pm 0,9$ & $39 \pm 2$ & 640 & 74 \\
\hline $\mathrm{OEU}_{2}$ & $87 \pm 1$ & $6_{4}^{\prime}, 1 \pm 0,8$ & $66,9 \pm 0,7$ & $62,9 \pm 1,0$ & $53 \pm 1$ & 628 & 81 \\
\hline $\mathrm{ODU}_{1}$ & $71 \pm 8$ & $63,9 \pm 0,4$ & $66,3 \pm 0,5$ & $64,8 \pm 0,9$ & $26 \pm 4$ & 627 & 68 \\
\hline $\mathrm{ODU}_{2}$ & $80 \pm 2$ & $62,3 \pm 1,3$ & $64,6 \pm 1,3$ & $65,2 \pm 1,3$ & $17 \pm 5$ & $61 / 4$ & 91. \\
\hline OETS $_{1}$ & $76 \pm 6$ & $67,2 \pm 0,7$ & $69,7 \pm 0,8$ & $62,0 \pm 1,6$ & $42 \pm 3$ & 661 & 71 \\
\hline $\mathrm{OETS}_{2}$ & $86 \pm 2$ & $66,1 \pm 0,8$ & $68,8 \pm 0,8$ & $62,5 \pm 0,7$ & $48 \pm 2$ & 649 & 77 \\
\hline \multicolumn{8}{|l|}{ Mais } \\
\hline $\mathrm{ME}_{2}$ & $59 \pm 3$ & $61,4 \pm 1,5$ & $63,7 \pm 1,5$ & $50,0 \pm 1,9$ & $34 \pm 3$ & 623 & 41 \\
\hline $\mathrm{MD}_{2}$ & $69 \pm 2$ & 58,5 土 0,8 & $60,1 \pm 0,7$ & $55,1 \pm 1,0$ & $12 \pm 1$ & 588 & 48 \\
\hline $\mathrm{MEU}_{1}$ & $73 \pm 2$ & 69,8 土 0,6 & $71,2 \pm 0,6$ & $66,7 \pm 0,9$ & $41 \pm 2$ & 696 & 75 \\
\hline $\mathrm{MEU}_{2}$ & $70 \pm 2$ & $70,6 \pm 0,7$ & $72,5 \pm 0,7$ & $74,8 \pm 0,7$ & $47 \pm 3$ & 708 & $12^{\prime}$ \\
\hline $\mathrm{MDU}_{1}$ & $87 \pm 1$ & $67,9 \pm 0,5$ & $69,2 \pm 0,5$ & $67,3 \pm 1,1$ & $29 \pm 2$ & 672 & 89 \\
\hline $\mathrm{MDU}_{2}$ & $72 \pm 1$ & $67,4 \pm 0,5$ & $68,8 \pm 0,5$ & $72,6 \pm 0,6$ & $32 \pm 2$ & 670 & 119 \\
\hline METS $_{1}$ & $83 \pm 1$ & $71,6 \pm 1,2$ & $73,0 \pm 1,1$ & $69,2 \pm 1,9$ & $38 \pm 5$ & 702 & 88 \\
\hline METS $_{2}$ & $73 \pm 1$ & $73,0 \pm 0,7$ & $74,2 \pm 0,8$ & $73,2 \pm 1,2$ & $48 \pm 2$ & 719 & 119 \\
\hline MDTS $_{1}$ & $91 \pm 1$ & $66,9 \pm 1,7$ & $68,2 \pm 1,7$ & $65,2 \pm 0,8$ & $26 \pm 4$ & 660 & 80 \\
\hline $\mathrm{MDTS}_{2}$ & $74 \pm 1$ & $70,9 \pm 0,7$ & $71,8 \pm 0,7$ & $72,4 \pm 1,1$ & $33 \pm 2$ & 689 & 121 \\
\hline
\end{tabular}

$1=$ récolte $1967 ; 2=$ récolte 1968

Les teneurs en MAD/kg MS des céréales seules sont évidemment faibles : 56 pour l'orge, et $4 \mathrm{I}$ pour le maïs sous forme ensilée, et respectivement $5 \mathrm{I}$ et 48 pour les formes déshydratées. Après supplémentation ces teneurs sont évidemment beaucoup plus fortes.

\section{5. - Utilisation métabolique de la matière azotée (tabl. 6, 7 et 8)}

Malgré le rationnement égalisé, certains impondérables (refus accidentels de nourriture, CUD et gain de poids dissemblables), n'ont pas permis de maintenir rigoureusement identiques pour tous les régimes, les niveaux énergétiques (MOD/ $\mathrm{P}^{0,75}$ ) et le rapport $\mathrm{NDa} / \mathrm{MOD}$, mais les différences notées sont très faibles et non significatives, en particulier entre régimes supplémentés en azote.

D'après les données du bilan, on note que :

a) Sans supplémentation azotée, les coefficients de rétention (CR) et d'utilisation pratique (CUP) des protéines propres de 1'orge sont respectivement de 47,5 et 25,6 pour la forme déshydratée contre 27,0 et 13,9 pour la forme ensilée. Ceux du maîs sont de 45 , $x$ et 24,9 pour la première forme de présentation, au lieu de 28 , I et I4,5 pour la seconde. Les différences entre traitements technologiques sont hautement 
TABLEAU 6

Données moyennes $\left( \pm s_{m}\right)$ du bilan azoté

\begin{tabular}{|c|c|c|c|c|c|c|c|c|}
\hline & \multicolumn{8}{|c|}{ Orge immature } \\
\hline & $\begin{array}{l}\text { MOD } \\
/ \mathrm{P}^{0,75}\end{array}$ & $\begin{array}{l}\text { NDa } \\
/ \mathrm{P}^{0,75}\end{array}$ & $\begin{array}{c}\text { NDa } \\
\text { /MOD }\end{array}$ & $\left|\begin{array}{c}\mathrm{N} \text { soluble } \\
\text { p. } 100 \\
\mathrm{~N} \text { total }\end{array}\right|$ & $\begin{array}{c}\mathrm{N} \text { bilan } \\
/ \mathrm{P}^{0,75}\end{array}$ & $\frac{\mathrm{I}-\mathrm{F}-\mathrm{U}}{\mathrm{I}-\mathrm{F}} \times 100$ & $\frac{\mathrm{I}-\mathrm{F}-\mathrm{U}}{\mathrm{I}} \times 100$ & $\begin{array}{l}\text { Poids } \\
\text { moy. } \\
(\mathrm{kg})\end{array}$ \\
\hline $\mathrm{OEU}_{1}$ & $\begin{array}{r}47,4 \\
\pm \quad 1,5\end{array}$ & $\begin{array}{r}0,90 \\
\pm 0,06\end{array}$ & $\begin{array}{r}1,79 \\
+0,12\end{array}$ & 52,0 & $\begin{array}{r}0,25 \\
\pm 0,06\end{array}$ & $\begin{array}{r}27,3 \\
\pm \quad 4,8\end{array}$ & $\begin{array}{r}17,9 \\
\pm \quad 3,2\end{array}$ & 255 \\
\hline $\mathrm{OEU}_{2}$ & $\begin{array}{r}54,6 \\
\pm \quad 0,5\end{array}$ & $\begin{array}{r}1,12 \\
\pm 0,06\end{array}$ & $\begin{array}{r}2,05 \\
\pm 0,04\end{array}$ & 47,8 & $\begin{array}{r}0,25 \\
+\quad 0,04\end{array}$ & $\begin{array}{r}22,1 \\
+\quad 3,2\end{array}$ & $\begin{array}{r}14,0 \\
\pm \quad 2,2\end{array}$ & 274 \\
\hline OETS $_{1}$ & $\begin{array}{r}49,8 \\
\pm \quad 3,2\end{array}$ & $\begin{array}{r}0,85 \\
\pm 0,05\end{array}$ & $\begin{array}{r}1,69 \\
\pm 0,07\end{array}$ & 44,0 & $\begin{array}{r}0,32 \\
\pm 0,05\end{array}$ & $\begin{array}{r}36,4 \\
+\quad 3,9\end{array}$ & $\begin{array}{r}22,5 \\
\pm \quad 2,4\end{array}$ & 271 \\
\hline OETS & $\begin{array}{r}55,8 \\
+\quad 1,4\end{array}$ & $\begin{array}{r}1,06 \\
\pm 0,03\end{array}$ & $\begin{array}{r}1,91 \\
\pm 0,03\end{array}$ & 32,6 & $\begin{array}{r}0,29 \\
\pm 0,02\end{array}$ & $\begin{array}{r}27,3 \\
\pm \quad 5,0\end{array}$ & $\begin{array}{r}17,1 \\
\pm \quad 1,0\end{array}$ & 263 \\
\hline $\mathrm{ODU}_{1}$ & $\begin{array}{r}44,8 \\
\pm \quad 4,1\end{array}$ & $\begin{array}{r}0,80 \\
+\quad 0,10\end{array}$ & $\begin{array}{r}1,75 \\
\pm 0,07\end{array}$ & 47,2 & $\begin{array}{r}0,37 \\
\pm 0,07\end{array}$ & $\begin{array}{r}45,6 \\
\pm \quad 3,0\end{array}$ & $\begin{array}{r}29,6 \\
\pm \quad 2,3\end{array}$ & 255 \\
\hline $\mathrm{ODU}_{2}$ & $\begin{array}{r}49,1 \\
\pm \quad 1,0\end{array}$ & $\begin{array}{r}1,16 \\
\pm 0,03\end{array}$ & $\begin{array}{r}2,37 \\
+0,03\end{array}$ & 35,3 & $\begin{array}{r}0,53 \\
\pm 0,03\end{array}$ & $\begin{array}{r}46,1 \\
+\quad 2,1\end{array}$ & $\begin{array}{r}30,0 \\
\pm \quad 1,3\end{array}$ & 259 \\
\hline $\mathrm{OD}_{2}$ & $\begin{array}{r}48,1 \\
\pm \quad 0,5\end{array}$ & $\begin{array}{r}0,76 \\
\pm 0,01\end{array}$ & $\begin{array}{r}1,57 \\
\pm 0,02\end{array}$ & 13,8 & $\begin{array}{r}0,36 \\
\pm 0,04\end{array}$ & $\begin{array}{r}47,5 \\
\pm \quad 4,2\end{array}$ & $\begin{array}{r}25,6 \\
\pm \quad 3,0\end{array}$ & 309 \\
\hline $\mathrm{OE}_{2}$ & $\begin{array}{r}53,3 \\
\pm \quad 0,6\end{array}$ & $\begin{array}{r}0,70 \\
+\quad 0,01\end{array}$ & $\begin{array}{r}1,31 \\
\pm \quad 0,03\end{array}$ & 36,9 & $\begin{array}{r}0,19 \\
\pm \quad 0,02\end{array}$ & $\begin{array}{r}27,0 \\
\pm \quad 3,8\end{array}$ & $\begin{array}{r}13,9 \\
\pm \quad 1,9\end{array}$ & 307 \\
\hline
\end{tabular}

$1=$ récolte $1967 ; 2=$ récolte 1968 NDa Azote digestible apparent

significatives $(\mathrm{P}<\mathrm{O}, \mathrm{OI})$ pour chacune des céréales en faveur de la déshydratation. Les protéines d'orge et de maîs ont une efficacité équivalente pour une même forme de présentation.

b) Avec supplémentation azotée, dans le cas de l'orge, les CR des régimes ODU, OE'TS et OEU sont respectivement de 45,8, 3I,9 et 24,9. L'écart en faveur de la forme déshydratée (ODU), est hautement significatif $(\mathrm{P}<0,0 \mathrm{I})$ par rapport à la forme ensilée complétée par l'urée (OEU) et significatif seulement $(\mathrm{P}<0,05)$ par rapport à la forme ensilée complétée par le tourteau de soja (OETS). D'autre part, le produit ensilé supplémenté par de l'azote de soja (OETS) est significativement meilleur $(\mathrm{P}<0,05)$ que celui supplémenté par de 1'azote uréique (OEU). Le CUP de ODU est aussi significativement $(\mathrm{P}<0,05)$ plus élevé $(29,8)$ que celui des deux autres régimes qui ne diffèrent pas significativement entre eux (OE'TS : I9,6 ; OEU : I6,0). Dans le cas du maïs, les CR et les CUP de la forme déshydratée supplémentée avec l'urée (MDU) ou le tourteau de soja (MD'TS) et ceux de la forme ensilée additionnée du tourteau se soja (METS) sont identiques. Les valeurs de ces trois régimes sont significativement plus élevées $(\mathrm{P}<0,05)$ que celles de la céréale ensilée supplémentée par de l'urée (MEU). Les CR respectifs sont de :37,3, 37,4, 37,7, contre 24,5 et les CUP de $26,0,25,5,26,8$ contre 17,3 . 
TABLEAU 7

Données moyennes $\left( \pm s_{m}\right)$ du bilan azoté

\begin{tabular}{|c|c|c|c|c|c|c|c|c|}
\hline & \multicolumn{8}{|c|}{ Épis entiers de maïs immature } \\
\hline & $\begin{array}{l}\text { MOD } \\
/ P^{0,75}\end{array}$ & $\begin{array}{l}\mathrm{NDa} \\
/ \mathrm{P}^{0,75}\end{array}$ & $\begin{array}{l}\text { NDa } \\
\text { /MOD }\end{array}$ & $\left|\begin{array}{c}\mathrm{N} \text { soluble } \\
\text { p. } 100 \\
\mathrm{~N} \text { total }\end{array}\right|$ & $\begin{array}{c}\mathrm{N} \text { bilan } \\
/ \mathrm{P}^{0,75}\end{array}$ & $\frac{\mathrm{I}-\mathrm{F}-\mathrm{U}}{\mathrm{I}-\mathrm{F}} \times 100$ & $\frac{\mathrm{I}-\mathrm{F}-\mathrm{U}}{\mathrm{I}} \times 100$ & $\begin{array}{l}\text { Poids } \\
\text { moy. } \\
(\mathrm{kg})\end{array}$ \\
\hline $\mathrm{MEU}_{1}$ & $\begin{array}{r}50,7 \\
+\quad 1,8\end{array}$ & $\begin{array}{r}0,88 \\
\pm 0,02\end{array}$ & $\begin{array}{r}1,75 \\
\pm 0,07\end{array}$ & 62,8 & $\begin{array}{r}0,20 \\
\pm 0,05\end{array}$ & $\begin{array}{r}22,7 \\
\pm \quad 5,3\end{array}$ & $\begin{array}{r}15,2 \\
\pm \quad 3,7\end{array}$ & 248 \\
\hline $\mathrm{MEU}_{2}$ & $\begin{array}{r}49,5 \\
+\quad 1,5 \\
\end{array}$ & $\begin{array}{r}1,38 \\
\pm 0,04 \\
\end{array}$ & $\begin{array}{r}2,80 \\
\pm 0,05 \\
\end{array}$ & 79,0 & $\begin{array}{r}0,36 \\
\pm 0,02 \\
\end{array}$ & $\begin{array}{r}26,2 \\
\pm \quad 1,6 \\
\end{array}$ & $\begin{array}{r}19,5 \\
+\quad 1,3\end{array}$ & 242 \\
\hline $\operatorname{METS}_{1}$ & $\begin{array}{r}59,1 \\
\pm \quad 1,5\end{array}$ & $\begin{array}{r}1,18 \\
+0,07\end{array}$ & $\begin{array}{r}1,99 \\
\pm 0,08\end{array}$ & 24,0 & $\begin{array}{r}0,47 \\
\pm 0,12\end{array}$ & $\begin{array}{r}39,8 \\
+\quad 9,6\end{array}$ & $\begin{array}{r}27,7 \\
\pm \quad 6,9\end{array}$ & 284 \\
\hline $\mathrm{METS}_{2}$ & $\begin{array}{r}52,5 \\
\pm \quad 0,5 \\
\end{array}$ & $\begin{array}{r}1,40 \\
+0,02 \\
\end{array}$ & $\begin{array}{r}2,66 \\
\pm 0,03\end{array}$ & $3 \frac{1}{4}, 4$ & $\begin{array}{r}0,50 \\
+0,0 \mathrm{k}\end{array}$ & $\begin{array}{r}35,6 \\
\pm \quad 2,3 \\
\end{array}$ & $\begin{array}{r}26,0 \\
\pm \quad 1,8\end{array}$ & 275 \\
\hline $\mathrm{MDU}_{1}$ & $\begin{array}{r}58,8 \\
\pm \quad 1,1\end{array}$ & $\begin{array}{r}1,2 / \mathbf{k} \\
\pm \quad 0,0^{\prime}\end{array}$ & $\begin{array}{r}2,10 \\
+0,03\end{array}$ & 45,2 & $\begin{array}{r}0,48 \\
\pm \quad 0,06\end{array}$ & $\begin{array}{r}39,5 \\
+\quad 5,7\end{array}$ & $\begin{array}{r}26,4 \\
\pm \quad 3,5\end{array}$ & 267 \\
\hline $\mathrm{MDU}_{2}$ & $\begin{array}{r}48,4 \\
\pm 00,7 \\
\end{array}$ & $\begin{array}{r}1,37 \\
+0,03 \\
\end{array}$ & $\begin{array}{r}2,82 \\
\pm 0,02 \\
\end{array}$ & 54,3 & $\begin{array}{r}0,48 \\
\pm 0,02 \\
\end{array}$ & $\begin{array}{r}35,2 \\
\pm \quad 1,0 \\
\end{array}$ & $\begin{array}{r}25,6 \\
\pm \quad 0,7\end{array}$ & 251 \\
\hline $\mathrm{MDTS}_{1}$ & $\begin{array}{r}60,1 \\
\pm \quad 0,8\end{array}$ & $\begin{array}{r}1,21 \\
\pm 0,0^{\prime}\end{array}$ & $\begin{array}{r}2,01 \\
\pm 0,06\end{array}$ & 8,21 & $\begin{array}{r}0,50 \\
\pm 0,07\end{array}$ & $\begin{array}{r}41,4 \\
\pm \quad 2,6\end{array}$ & $\begin{array}{r}26,9 \\
\pm \quad 1,6\end{array}$ & 287 \\
\hline $\mathrm{MDTS}_{2}$ & $\begin{array}{r}51,7 \\
\pm \quad 0,8 \\
\end{array}$ & $\begin{array}{r}1,1^{\prime} \mathbf{k} \\
\pm 0,04 \\
\end{array}$ & $\begin{array}{r}2,78 \\
\pm 0,05 \\
\end{array}$ & 18,09 & $\begin{array}{r}0,48 \\
\pm 0,03 \\
\end{array}$ & $\begin{array}{r}33,5 \\
\pm \quad 2,4 \\
\end{array}$ & $\begin{array}{r}24,1 \\
\pm \quad 1,6 \\
\end{array}$ & 278 \\
\hline $\mathrm{MD}_{2}$ & $\begin{array}{r}40,7 \\
+\quad 1,1\end{array}$ & $\begin{array}{r}0,53 \\
+0,02\end{array}$ & $\begin{array}{r}1,30 \\
\pm 0,03\end{array}$ & 17,5 & $\begin{array}{r}0,24 \\
+0,01\end{array}$ & $\begin{array}{r}45,1 \\
+\quad 1,3\end{array}$ & $\begin{array}{r}24,9 \\
\pm \quad 1,1\end{array}$ & 257 \\
\hline $\mathrm{ME}_{2}$ & $\begin{array}{r}50,7 \\
+\quad 1,8\end{array}$ & $\begin{array}{r}0,39 \\
\pm 0,03\end{array}$ & $\begin{array}{r}1,06 \\
\pm 0,03\end{array}$ & 69,9 & $\begin{array}{r}0,12 \\
\pm 0,02\end{array}$ & $\begin{array}{r}28,1 \\
\pm \quad 3,6\end{array}$ & $\begin{array}{r}1 /, 5 \\
\pm \quad 2,3\end{array}$ & $2 / 3$ \\
\hline
\end{tabular}

$1=$ récolte $1967 ; 2=$ récolte $1968 *$ NDa - Azote digestible apparent

La seule comparaison des CUP fait ressortir nettement les différences d'efficacité des régimes en fonction du traitement et de la source azotée.

Traitement. - Que la céréale soit non supplémentée ou supplémentée en urée, la forme déshydratée est fortement supérieure à la forme ensilée. En l'absence d'urée, cette supériorité est de $+84 \mathrm{p}$. Ioo avec l'orge et de $+72 \mathrm{p}$. Ioo avec le maïs; en présence d'urée, les pourcentages respectifs sont de +87 et $+50(\mathrm{P}<0$,or $)$. Par contre, les deux formes de présentation de la céréale procurent une efficacité identique du moins dans le cas du maîs (orge déshydratée + tourteau de soja n'a pas été expérimenté) lorsque la supplémentation est faite avec du tourteau de soja.

Source azotée. - Le soja est non significativement $(+24 \mathrm{p}$ IOo) plus efficace que l'urée en présence d'orge et, significativement $(+54 \mathrm{p}$ I00) e n présence de maïs lorsque la céréale est sous forme d'ensilage. Les deux sources ont une valeur identique lorsque la céréale (maïs) est deshydratée. 
TABLEAU 8

Valeurs moyennes du bilan azoté

\begin{tabular}{|c|c|c|c|c|c|c|c|}
\hline \multirow{7}{*}{\begin{tabular}{l}
\multicolumn{1}{c}{ Orge } \\
MOD/P0 \\
NDa/MOD \\
Nsol/Ntot \\
CR \\
CUP
\end{tabular}} & \multicolumn{2}{|c|}{ Céréales non supplémentées } & \multicolumn{5}{|c|}{ Céréales supplémentées en azote } \\
\hline & $\mathrm{OE}^{*}$ & OD* & OEU & OETS & ODU & & \\
\hline & 53,3 & 48,1 & $50,6 a b$ & $52,8 a$ & $47,0 b$ & & (1) \\
\hline & 1,31 & 1,57 & $1,91 a b$ & $1,80 a$ & $2,06 b$ & & (2) \\
\hline & 37,0 & 14,0 & 50,0 & 38,0 & 41,0 & & \\
\hline & 27,0 & 47,5 & $24,9 a$ & $31,9 b$ & $45,8 c$ & & (3) \\
\hline & 13,9 & 25,6 & $16,0 a$ & $19,8 a$ & $29,8 b$ & & $(4)$ \\
\hline Maïs & ME* & $M D^{*}$ & MEU & METS & MDU & MDTS & \\
\hline $\mathrm{MOD} / \mathrm{P}^{0^{\prime} 75}$ & 36,9 & 40,7 & $50,1 a$ & $55,8 b$ & $53,6 b$ & $55,9 b$ & (5) \\
\hline $\mathrm{NDa} / \mathrm{MOD}$ & 1,06 & 1,30 & $2,50 a$ & $2,43 a$ & $2,58 a$ & $2,48 a$ & (6) \\
\hline Nsol/Ntot & 70,0 & 18,0 & 71,0 & 29,0 & 50,0 & 13,0 & \\
\hline $\mathrm{CR}$ & 28,1 & 45,1 & $2 t, 5 a$ & $37,7 b$ & $37,3 b$ & $37,4 b$ & (7) \\
\hline CUP & 14,5 & 24,9 & $17,3 a$ & $26,8 b$ & $26,0 b$ & $25,5 b$ & (8) \\
\hline
\end{tabular}

* Toutes différences hautement significatives $\mathrm{P}<0,01$ entre les deux traitements non supplémentés. Les données désignées par la même lettre ne sont pas significativement différentes. (8) $\mathrm{P}<0,01$.

Seuil de signification des différences : pour (1), (3) $a-b,(5) \mathrm{P}<0,05$; pour (2), (3) $b-c, \quad(4),(7)$,

\section{(8) $P<0,01$,}

III.

DISCUSSION

Les résultats rapportés intéressent les régimes globaux tels qu'ils ont été ingérés, c'est-à-dire : céréales enrichies soit en cellulose setlement, soit simultanément en cellulose et en $\mathrm{N}$.

\section{I. - Valeur alimentaire}

Certains auteurs ont observé que, par rapport au grain mûr non traité, la cuisson de l'orge (Kojrms et al., 1968) ou la gélatinisation par autoclavage du maîs (MUDD et PERRY, I 669 ) entraine une baisse de la digestibilité de la plupart des composants majeurs de la ration.

Il n'est cependant pas certain que, dans nos essais, la moins bonne digestibilité de la matière organique et surtout de la cellulose Weende observée avec la céréale immature déshydratée soit due au traitement thermique. Il est vraisemblable que 1e conditionnement (broyage fin et agglomération) appliqué après déshydratation en soit plutôt le facteur déterminant. On sait en effet que par rapport à la forme longue, ce mode de conditionnement d'un aliment active le transit digestif, et abaisse sensiblement la digestibilité de la matière organique plus spécialement celle des constituants membranaires.

I a baisse des CUD observée par suite de la déshydratation et de la condensation entraîne forcément celle de la richesse en énergie digestible. 
Si l'on compare les teneurs en MOD des deux modes de présentation de la céréale non supplémentée, l'on constate que les valeurs de l'orge et du maïs déshydratés sont respectivement de $-9 \mathrm{p}$. Ioo et de $-6 \mathrm{p}$. Ioo inférieures à celles des mêmes produits ensilés.

Si l'on calcule les valeurs d'énergie nette (UF (1)), mode d'expression habituel des tables d'alimentation, les écarts sont plus élevés encore en faveur des produits ensilés : - I4 p. Ioo pour l'orge $\{0,63$ contre $0,73 \mathrm{UF} / \mathrm{kg} \mathrm{MS}\rangle$ et $-9 \mathrm{p}$. roo pour le mais $(0,65$ contre $0,72 \mathrm{UF} / \mathrm{kg} \mathrm{MS})$.

Quel que soit le mode d'expression admis, la supplémentation des céréales en azote uréique qui améliore le rapport azote/énergie de la ration, réduit non seulement ces écarts mais encore améliore la valeur nutritive de la forme ensilée, du moins celle du maïs.

Cependant les écarts en question pourraient bien n'être qu'apparents puisque selon BLAXTER et al. (1956), la baisse de digestibilité provoquée par le broyage et la condensation serait compensée par une réduction des autres pertes métaboliques et de ce fait, il n'y aurait pratiquement pas de différence d'énergie nette entre forme longue et forme condensée d'un fourrage.

\section{2. - Efficacité azotée}

L'amélioration du CUD de l'azote des régimes après enrichissement soit par de l'urée soit par du soja, est vraisemblablement liée au fait que :

a) 1'azote de ces compléments est en soi plus digestible que celui de la céréale immature ;

b) la digestibilité apparente de l'azote est d'autant plus faible que le régime est pauvre en azote, en raison de la part relative plus importante de la fraction azotée endogène.

I'action favorable de la forme déshydratée sur l'efficacité de $\mathrm{N}$ uréique est beaucoup plus notable avec l'orge qu'avec le maïs. L,es différences dans les niveaux d'énergie $\left(\mathrm{MOD} / \mathrm{P}^{0,75}\right)$ et les rapports protéines/calories (NDa/MOD) des régimes n'expliquent pas cet effet, car d'une manière générale les premiers sont moins élevés, et les seconds plus élevés avec les régimes ensilés. Or, les protéines pouvant être partiellement employées pour compenser le déficit énergétique (BALCH et BINES, I968) chez des animaux soumis à une alimentation énergétique limitée, comme les nôtres, cela aurait dû entraîner logiquement des bilans d'azote plutôt défavorables à la forme déshydratée alors que l'on observe l'inverse.

On est conduit dès lors à supposer l'intervention d'au moins deux autres facteurs : a) la teneur des régimes en azote soluble et (ou) b) 1'état structural de l'amidon.

a) Intervention de la fraction azotée soluble.

Ce facteur a sans doute du jouer, dans le cas des céréales consommées seules : la concentration en azote soluble facilement attaquable par les désaminases micro-

(1) Formule de calcul des UF/kg MS $=\frac{(\text { MOD }+ \text { I,25 MGD) } 3,65-I \text { ooo }}{\text { I } 883_{3}}$ 
biennes du rumen est de loin plus élevée dans la forme ensilée que dans celle déshydratée $(2,5$ fois dans l'orge et 4 fois dans le maiss). Ces différences très marquées peuvent donc y modifier l'utilisation métabolique de l'azote, dans un sens favorable à la déshydratation.

L,es régimes de céréales ensilées complétés par de l'urée étant de même caractérisés par une très grande richesse en $\mathrm{N}$ soluble (OEU 50 p. IOO ; MEU $7 \mathrm{I}$ p. IOo $\mathrm{N}$ total) cela pourrait également expliquer leurs CUP extrêmement bas et voisins de cetux des céréales ensilées non supplémentées.

Par contre, la teneur en $\mathrm{N}$ soluble ne semble pas avoir été la cause déterminante dans le cas des trois autres régimes supplémentés en protéines. Le régime ODU relativement riche en N soluble ( 35 à $47 \mathrm{p}$. Ioo) présente le CUP le plus élevé alors que celui du régime OETS qui en contient pratiquement autant (33 à 44 p. I00) est nettement plus faible. Avec une teneur moyenne de $50 \mathrm{p}$. roo de $\mathrm{N}$ soluble, le régime de MDU a le même CUP que les régimes METS et MDTS qui n'en renferment respectivement que $29 \mathrm{p}$. Ioo et $\mathrm{I} 3 \mathrm{p}$. Ioo.

\section{b) Intervention des modifications induites dans l'amidon de la céréale par l'ensilage et la déshydratation.}

Si la teneur originelle en amidon total est à peine modifiée par le traitement, celle en amidon facilement attaquable par l' $\alpha$-amylase l'est, par contre, profondément (tab1. 4). Après ensilage, cette dernière fraction est abaissée en moyenne de $40 \mathrm{p}$. Ioo dans l'orge, et de 45 p. Ioo dans le mais, par suite de sa dégradation en acides organiques, alors qu'elle est augmentée considérablement après déshydratation $: 4,5$ fois dans l'orge et 2,6 fois dans le maïs.

En raison de ces modifications, le rapport entre amidon facilement attaquable et urée incorporée (tabl. 4). déjà faible à l'origine $(3,5$ à 5 dans 1'orge et 0,9 à 2 dans le maïs) diminue encore davantage dans l'ensilage (respectivement 2,5 et 0,4 à 0,7$)$ alors qu'il augmente énormément dans les produits déshydratés, où il est en moyenne 5 fois plus élevé dans 1'orge (20) que dans le maïs (4). De plus, le traitement thermique accélère beaucoup la rapidité de la dégradation enzymatique de cette fraction (fig. I).

Tout se serait donc passé comme si la céréale déshydratée offrait un contenu glucidique apte à fournir à la micropopulation de la panse davantage d'énergie rapidement disponible après déshydratation qu'après ensilage. Ce qui aurait permis à cette microflore d'incorporer dans sa propre synthèse d'acides aminés, des quantités plus fortes d'azote ammoniacal engendré par le régime et plus spécialement par l'urée, et expliquerait ainsi les bilans azotés très favorables obtenus avec la forme déshydratée. Lue phénomène est en outre beaucoup plus accusé avec l'orge qu'avec le mais, supplémenté en urée.

L'étude cinétique comparée in vivo de la libération des glucides alcoolo-solubles $\left(\mathrm{G}_{1}-\mathrm{G}_{14}\right)$ dans le rumen du mouton à partir d'une même orge immature déshydratée ou ensilée, montre effectivement que la première forme procure à la micropopulation du rumen, l'énergie qui lui permet de capter au fur et à mesure de sa formation l'ammoniaque engendrée par l'hydrolyse de l'urée; alors qu'avec la forme ensilée, cette micropopulation n'a à sa disposition au moment du pic ammoniacal que très peu de ces glucides dont la concentration n'apparaît importante 
qu'au moment ( $5^{\mathrm{e}}$ heure post-prandiale) où une large part de $\mathrm{N}$ ammoniacal a déjà quitté la panse et que sa concentration est revenue au niveau initial (DURAND et al., I970).

Un traitement hydrothermique approprié d'une céréale immature pourrait donc rendre son amidon plus accessible à la dégradation microbienne dans la panse et la vitesse de libération de son énergie concomitante sinon parallèle à celle de l'uréolyse de l'urée ajoutée.

Des recherches contemporaines (HELMER et al., I970; LANG et al., r970) montrent également que le traitement thermique du grain mûr de maîs ou de sorgho par extrusion sous pression, additionné d'urée avant ou après, améliore fortement la sensibilité amylolytique de l'amidon, et stimule, du moins in vitro (HELMER et al., I970) la protéosynthèse bactérienne à partir d'azote uréique.

L'incorporation maximum de $\mathrm{N}$ uréique dans les protéines microbiennes du rumen serait done plutôt tributaire de la quantité totale d'amidon facilement et rapidement attaquable par les amylases bactériennes du milieu que de celle de glucides totaux fermentescibles.

Ia moins bonne réponse du maïs que celle de l'orge à l'urée pet1t s'expliquer par d'autres processus :

- les différents types d'amidon fermentent avec des intensités différentes dans la panse ; celui de l'orge notamment fermente plus complètement que celui de maïs. En effet, près de 90 p. Ioo de l'amidon d'orge ingéré disparaissent durant le passage dans le rumen alors que près de $30 \mathrm{p}$. roo d'amidon de maîs quittent cet organe digestif sans avoir été dégradés par sa micropopulation (CARR et al., I966; TUCkER et al., I968; Orskov, I969; THIVEND et JoURnEk I968 et I970) ;

- fermentation glucidique et croissance bactérienne sont fortement imbriquées dans le rumen. Les deux processus sont simultanés et la quantité de glucides qui peut y être utilisée dépend de la synthèse de nouvelles cellules microbiennes qui remplacent celles qui le quittent. Si les glucides sont le substrat limitant pour la synthèse bactérienne, la quantité de protéines synthétisées par unité de glucides ingérées est d'autant plus faible que la fraction de glucides échappée à l'attaque bactérienne dans le rumen est plus importante, car elle est perdue pour la protéosynthèse microbienne (ORsKov, I969).

Sur le plan zootechnique, l'on peut admettre, d'après nos données, que l'efficacité de l'urée comme source de complémentation azotée de la céréale immature est très nettement déterminée par la forme de présentation de celle-ci. Elle est égale (maïs) et serait même supérieure (orge) à celle du tourteau de soja lorsque la céréale est traitée par déshydratation, mais sensiblement inférieure lorsqu'elle est ensilée. Dans ce dernier cas, l'urée ne semble pas pouvoir remplacer le tourteau de soja, alors qu'elle peut le faire certainement dans le premier. La supériorité de la déshydratation semblerait due en premier lieu aux modifications favorables induites par le traitement hydrothermique dans la structure de l'amidon. Ces modifications, moins prononcées dans le maîs que dans l'orge, rendent l'énergie davantage disponible pour la micropopulation du rumen. Elles assurent une transformation substantielle du $\mathrm{NH}_{3}$ provenant de l'urée en protéines microbiennes. Chez le ruminant, le facteur limitant majeur de l'utilisation de $\mathrm{N}$ uréique reste sans doute la possibilité de faire coïncider les intensités de libération de $\mathrm{NH}_{3}$ et d'énergie dans la panse.

L'intérêt agronomique de la récolte de céréales à un stade immature n'étant Annales de Zootechnie. - I97I. 
pas évident, vu les conditions climatiques prévalant dans la majeure partie de la France d'une part, et l'action bénéfique du traitement thermique d'un grain immature sur l'efficacité de l'urée paraissant certaine d'autre part, il est opportun de savoir :

- si semblable traitement appliqué au grain mûr réhumidifié procurerait de résultats équivalents à ceux obtenus avec la céréale immature ;

- quelles sont les céréales les plus justifiables d'un tel traitement;

- si ce procédé est économiquement intéressant au plan zootechnique.

Les deux premiers points sont en cours d'étude.

Rę̧u pour publication en décembre 1970.

\section{REMERCIEMENTS}

Les auteurs expriment leurs vifs remerciements à A. GuIlbot et Th. Cerning (Station de Biochimie et Physico-Chimie des céréales I. N. R. A.) pour avoir bien voulu effectuer les tests d'amylolyse.

\section{SUMMARY}

\section{EFFECT OF CONSERVATION (SILAGE OR DEHYDRATION) OF IMMATURE CEREALS (BARLEY, MAIZE) \\ UPON THEIR ATRITIVE VALUE AND THE METABOLIC EFFICIENCY OF UREA IN THE GROWING STEER}

44 steers and two immature cercals (barley and maize) were used during 4 successive experiments to study the effect of the mode of conservation of these cereals (silage, dehydration) on :

a) the digestibility and nutritive value of these feeds,

b) the utilization of their proteins,

c) the utilization of urea nitrogen in comparison with that of soybean oil meal.

Two successive crops ( 1967 and 1968) of barlcy (Rika variety), cut $15-30 \mathrm{~cm}$ below the ear, and whole maize cobs $(I . N . R . A$. variety 260$)$ were harvested $2-4$ weeks before normal maturity of the grain and treated either by silage under partial vacuum, or by dehydration in a low temperature dryer ( $120-\mathrm{I} 30^{\circ} \times 30-40 \mathrm{mn}$ ). The measurements of the digestibility and nitrogen retention were carried out according to a latin square design or a design of comparable blocs (table $\mathbf{r}$ ). The following results were obtained :

a) dehydrated and condensed product has a lower digestibility than the chopped and ensiled product; the same holds probably true as regards the energy value (table 5).

b) the nitrogen retention coefficient ( $\mathrm{N}$ retained/N digested( (CIR) and protein efficiency coefficient ( $\mathrm{N}$ retained/N ingested) (CUP) are significantly higher for the dehydrated than for ensiled products. The superiority of the dehydrated product may be imputable to its very low content of soluble nitrogen (tables 6-7-8).

c) the metabolic utilization of urea nitrogen is significantly improved when used as a supplement for the dehydrated cereal : it is equal (maize) and even higher (barley) than that of the soybean nitrogen. $6-7-8)$.

In the case of ensiled cercals, urea is significantly less efficient than soybean oil meal (tables

d) the highly significant positive action of dehydration upon the utilization of urea nitrogen would be related to the important increase of the amylolytic solubility ( $\alpha$-amylase) of the starch induced by heat treatment (table 4 and fig. I).

The nutritional and technical signification of the results obtained is thoroughly discussed. 


\section{RÉFÉRENCES BIBLIOGRAPHIQUES}

Balch C. C., 13ines J. A., I968. The effect on nitrogen retention by growing heifers of protein and non protein nitrogen fed in isocaloric rations. Proc. Nutr. Soc., 27, I4 A.

Blaxter L., Grainam N. Mac., 1956. The effect of the grinding and cubing on the utilization of the energy of dried grass. J. Agric. Sci., 47, 207-217.

Bloomfield R. A., Garner G. 13., Muhrer M. E., ig6o. Kinetics of urea in sheep. J. Anim. Sci., 19, 1248 .

Bloomfield R. A., Wilson R. P., Thompson G. 13., ig64, Influence of energy levels on urea utilization. J. Anim. Sci., 23,868.

Chalmers M. I., I969. Urea and other substitutes for natural proteins in the nutrition of livestock. Symposium de l'industrie européenne de l'alimentation animalc, octobre 1969 (Hoffmann-Laroche, Bâle, Suisse).

Charlet-Léry, G. Tisserand J. L., Zelter S.-Z., ig69. Valeur nutritive des céréales immatures (orge et maîs) cnsilées ou déshyclratées. A, F. Z. Prodtution des ruminants, 2, Comm. 28. I-28.9.

Durand M., I97o. Influence du traitement technologique de l'aliment énergétique (orge immature) sur le métabolisme de ses glucides et de l'urée dans le rumen du mouton. C.R. Acad Sci. (217, Serie D, 2I67-2170).

Drck G. W., I963. Qualitative and quantitative studies of the flow of digesta from the abomasum of sheep Thesis university of Manitoba (Winnipeg).

Gouet P. H., Riouy., Fatianoff N., r97i. Conservation par ensilage ou deshydration d'une orge et d'un maïs immaturs. Ann. Zootech. 20,3 (sous presse).

Guibot A., Mrrcier Ch., I962. Répercussion sur la digestibilité de l'amidon des modifications de sa structure physico-chimique au cours de ses transformations technologiques. Ind. Agric. Alim., 79 , I I, 939-947.

Helmer L. G., Bartley E. E., Deyoe C. W., Meyer R. M., PFost H. B., ig7o. Feed Processing V. Effect of an expansion-processed mixture of grain and urea (starea) on nitrogen utilization in vitro. J. Dairy Sci., 53, 330-335.

Hosinno S., Sarijmaru K., Morimoto K., ig66. Ammonia anabolism in Ruimnants. J. Dairy Sici., 12, I523-I 528 .

Hoover W. H., Kesler E. M., Flipse R. J., 1963. Carbon sources for in vitro protein synthesis by rumen bacteria. J. Dairy Sci., 46, 733-729.

Hungate R. E., I966. The rumen and its microbes Acad. Press., New York.

Kamzoka K., Morimoto II., 1962. Studies on the digestion in ruminants. Bull. Nat. Inst. Agr. Sci., Séries G 2I, I89 et I95.

KARR M. R., Little C. O., Mitchell G. E., I966. Starch dissappearance from different segments of the digestive tracts of steers. J. Anim. Sci., 25, 652-654.

Kojima Y., Nakai S., Kawashima R., Uesakas., I968. Effect of cooked barley or milo mixed to the ration on its digestibility. Japan J. Zoot. Sci., 39, 543-548.

LEwis D., r96I. Digestive physiology and nutrition of the ruminant (Butterworths, London), 297.

Liang Y. T., Morrill J. L., Anstaett 1.. R., Dayton A. D., Prost H. B., I97o. Effect of pressure moisture and cooking time on susceptibility of corn and sorghum grain starch to enzymatic attack. J. Dairy Sci., 53, 336-34I.

Mclarren G. A., Anderson G. C., Martin W. G., Cooper W. K., I961. Fixation of ammonia nitrogen by rumen mucosa. J. Anim. Sci., 20, 942.

Mclarren G. A., Anderson G. C., Tsai L. J., Barth K. M., i965. Level of readly fermentable carbohydrates and adaptation of lambs to all-urea supplemented rations. J. Nutr., 87, 33 I-336.

MöLleR P. D., I967. Untersuchung über dïe Harnstoff Verwertung während der Lactation. Dissertation Göttingen. Max Plank Inst Tierz. u. Tierernähr 32.

Möller P. D., 1968. Der Wert der Rohproteins für die Bedarfsdeskung bei Milchproduktion. 2. Harnstoffverwertung. Z. Tierphysiol. Tierernähr. Futtermittelk., 23, 224-239.

Mudd C. A., Perry T. W., I969. Raw cracked vs expanded gelatinized corn for beef cattle. J. Anim. Sci., 28, 822-826.

Orskov E. R., I969. Post ruminal digestion of carbohydrates : extent and implications. Rev. cubana Cienc. Agri, 3, I-I3.

Thivend P., Journet M., I968. Utilisation digestive de l'amidon de l'orge chez le ruminant. Ann. Biol. anim. Bioch. Biophys., 8, 449-45I.

Thivend P., Journiet M., 1970. Utilisation digestive de l'amidon du mais chez le ruminant. Ann. Biol. anim. Bioch. Biophys., 10, 323-326. 
Tucker R. E., Mitchell G. E., Little C. O., I968. Ruminal and post-ruminal starch digestion un sheep. J. anim. Sci., 27, 824-826.

Zelter S.-Z., Charlet-Léry G., Tisserand J. L., ig69. Influence de la forme de présentation (ensilage, déshydratation) d'une céréale immature sur l'efficacité métabolique de l'urée chez le taurillon en croissance in tensive. A.F.Z. Production des ruminants, 2, Comm. 30.I-30.II.

Zelter S.-Z., Charlet-Lery G., I969. Forme de présentation de l'aliment énergénique (orge immature) et taux de retention de N-ureique chez le taurillon en croissance. C. R. Acad. Sci. (Paris), Série D. 269, I007-IoIo. 